\title{
Treatment of acute coronary syndromes: have we reached the ceiling?
}

\author{
Lars Wallentin
}

Non-ST elevation acute coronary syndrome (ACS) is currently the most common reason for admission to coronary care units. For roughly the past 15 years, combined platelet inhibition and coagulation activation has been a mainstay of treatment. In the 1980s, the first-generation antithrombotic drugs-aspirin and intravenous unfractionated heparin-halved event rates compared with placebo. In the 1990s, use of the second-generation platelet inhibitory agents, glycoprotein Ilb/llla inhibitors and oral clopidogrel, further reduced event rates by 20-30\%. Subcutaneous low-molecular-weight heparin also proved at least as effective as unfractionated heparin, and was simpler to use. Finally, several prospective randomized trials have demonstrated that, in addition to these antithrombotic medications, routine early coronary angiography and, if appropriate, revascularization reduces mortality and morbidity and improves quality of life without added costs.

The use of combined pharmacologic therapy and early revascularization has dramatically changed the natural course of ACS. In clinical trials, acute-phase (1 week) mortality is now reported as being only $2 \%$ and the reinfarction rate $2-4 \%$. Given that new treatments usually provide only $15-20 \%$ additional relative benefit, it is questionable whether an absolute therapy benefit of $0.5 \%$ is clinically relevant and cost effective. Have we, therefore, reached a ceiling in the early management of ACS patients? The focus of most trials remains on the early in-hospital phase of ACS; several current largescale trials are evaluating glycoprotein Ilb/Illa inhibitors, intravenous direct thrombin inhibitors and subcutaneous factor Xa inhibitors, compared with the standard regimen. Early invasive procedures are, however, still associated with a risk of complications, which might be reduced by more appropriate adjuvant

\section{We have not \\ yet reached \\ the ceiling \\ in the acute \\ or long-term \\ treatment of \\ ACS. By 2010 \\ we should have \\ seen several \\ improvements \\ in the \\ management \\ of this \\ condition.}

\section{$L$ Wallentin is the \\ Director of the \\ Uppsala Clinical \\ Research Centre, \\ University Hospital, \\ Uppsala, Sweden.}

Competing interests

The author has declared

competing interests; go to

the online article for details.

www.nature.com/clinicalpractice doi:10.1038/ncpcardio0153 treatment and better timing of the procedures. In addition, although event rates are low in the average population, several high-risk groups still have a potentially poor prognosis, such as elderly patients, or those with diabetes, renal dysfunction, myocardial dysfunction, previous coronary artery bypass grafts and other comorbidities. This might be improved by better, individually tailored early management.

In an average ACS population, mortality after 1 year is $3-4 \%$ and the frequency of nonfatal myocardial infarction is $6-8 \%$. Thus, over 1 year, a new strategy with a relative treatment benefit of 15-20\% will lead to at least 1.5-2.0\% absolute benefit. Substantial improvements on the current long-term antithrombotic treatment, aspirin and 3-12 months of clopidogrel, are still possible. Some potential new treatments have recently emerged. In a large-scale, phase II trial, 6 months of treatment with a combination of aspirin and the first oral thrombin inhibitor was associated with a $25 \%$ reduction in event rate. In addition, two new ADP inhibitors, which seem to provide more rapid and effective platelet inhibition than clopidogrel, are currently entering large-scale, phase II and III trials. Finally, some trials have indicated that early lowering of LDL cholesterol and C-reactive protein levels with statin therapy further reduces event rates. Improved lipidlowering and anti-inflammatory therapies have, therefore, become new areas of investigation in the long-term prevention of new events.

Thus, we have not yet reached the ceiling in the acute or long-term treatment of ACS. By 2010 we should have seen several improvements in the management of this condition. A ceiling might, however, be reached when our improved understanding of the underlying pathophysiology has led to clinically useful methods to permanently stabilize, reverse and prevent underlying atheroinflammatory disease. 